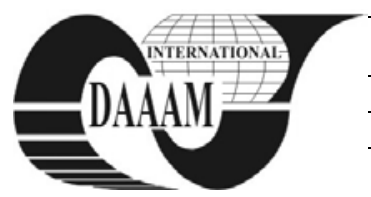

\title{
POWER SPECTRUM OF THE COMBINED DYNAMIC SYSTEMS
}

\author{
STREMY, M[aximilian] \& BEZAK, T[omas]
}

\begin{abstract}
In the article there are mentioned combined discrete dynamic systems and analysis of their power spectrum especially aimed to the event activated part of the system. Combined dynamic systems, also called hybrid systems are the fusion result of the time-driven and event activated systems. System consists of a combination of cyclically recurring processes in each period of processing and the stochastically emerging events. Power spectrum determination, described in the article, assumes stochastic events generation corresponding to the Normal probability distribution. The power spectrum of stochastic events generated by the Normal Poisson distribution in the combined systems is created using the theory of white noise.
\end{abstract}

Key words: normal distribution, stochastic events, combined dynamic systems, power spectrum, white noise

\section{INTRODUCTION}

Combined systems constist of two discrete systems parts: discrete time-dynamic-activated systems, as well as discrete events systems. Combined dynamic systems, also called hybrid systems are the fusion result of time-driven and event activated systems. In case that notion "Hybrid system" is used in connection between distributed control system and programmable logical controllers, neural networks, genetic algorithms and fuzzy logics, or combination of electric and mechanical power units (Ždánsky, 2009), for better prediction and better identification the concept of "Combined dynamic system" was introduced.

Generally, $T_{O}$ sampling period in dynamic systems is considered as constant and includes only the $T_{R}$ time required to process all the necessary control and cyclically recurring processes in the system. In contrast, the combined dynamic systems contemplate the sampling period, regardless of whether it is constant or variable, extended by the time constant $T_{P}$ needed to execute random events in the system (Fig. 1).

Event component of the sampling period significantly affects the behavior of the entire system. It affects controllability, stability and overall dynamic properties of the combined systems that are designed by ratio of events and timecontrolled discrete systems. Time-controlled part of the combined discrete systems is realized by a constant cyclic monitoring, processing and evaluation of inputs and states of the system. Event part is in automated control systems

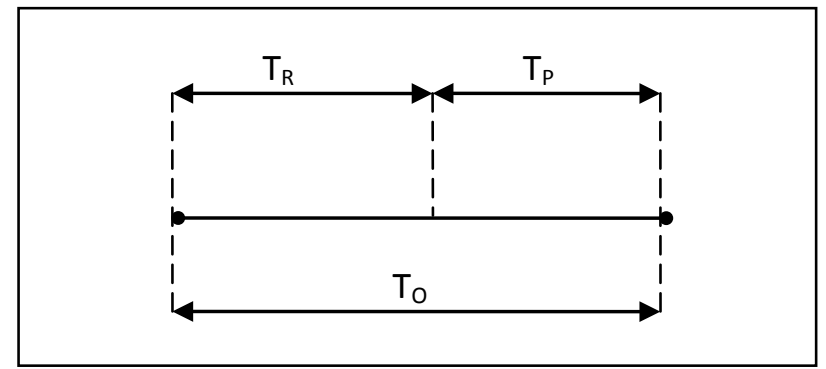

Fig. 1. Sampling Period $T_{O}$ in combined dynamic systems implemented by service interruptions, called upon the occurrence of any of the events. A particular problem in such systems appears to be how to determine the impact of stochastically changing event component of their dynamic properties and quality control (Strémy, 2010).

\section{NORMAL PROBABILITY DISTRIBUTION OF EVENT-TIME CONSTANT}

Normal probability distribution predicts balanced arising events distribution along the average value $T_{a}$ calculated from the equation

$$
T_{a}=\frac{T_{c}}{N},
$$

where $T_{c}$ is the time required to service all existing interrupts and $N$ is the number of interruptions occurring in a given cycle. Root mean square (rms) deviation from the mean value of $T_{a}$ is then determined by the relationship

$$
\sigma=\sqrt{\frac{\sum_{i=1}^{n}\left(x_{i}-\mu\right)^{2}}{n-1},}
$$

where $\mu=T_{a}$ - average time needed to handle new events.

Using the rms value criteria of randomly generated interrupts could be determined. This means how likely the events that occur in the sampling period of the combined dynamic system will be executed. The size of the sampling period corresponds to the time mean value $\mu$ in this case. The probability that the events in this interval extended / shortened by the time, which is the rms value equals to:

- $68,3 \%$ - in the $\mu+-\sigma$,

- $95,6 \%$ - in the $\mu+-2 \sigma$,

- $99,7 \%$ - in the $\mu+-3 \sigma$.

Probability density distribution $f(t)$ is the derivation of the distribution function $F(t)$. By this it is possible to determine the probability of particular events generation $N$ as well as the probability of this events creation amount in time interval $\Delta t$ (Banks, 2001):

$$
f(t)=\frac{d F(t)}{d t} \approx \frac{n_{x}(t+\Delta t)-n_{x}(t)}{N \Delta t} .
$$

Probability density distribution $\mathrm{f}(\mathrm{t})$ at time $\mathrm{t}$ has the form: 


$$
f(t)=\frac{1}{\sigma \sqrt{2 \pi}} e^{-\frac{(x-\mu)^{2}}{2 \sigma}},
$$

while its maximum value corresponds to the function $f(t)$ at time $T_{a}$

$$
f\left(T_{a}\right)=\frac{1}{\sigma \sqrt{2 \pi}}
$$

Time constant $T_{p}$, in the combined system may be equal to:

(1) the mean of the time needed to handle new events => $T_{p}=T_{a}$,

(2) the mean of the time needed for handling events generated enlarged / reduced by the value $\Delta=>T_{p}=T_{a}+-\Delta$,including time needed to handle events with a fixed probability percentage (e.g. $+\sigma$ means probability around $74 \%$ )

(3) the mean of the time needed to handle new events and three times the rms value of $T_{p}=T_{a}+3 \sigma$. This value includes handling of all events in the cycle with a probability of $99.7 \%$.

\section{POWER SPECTRUM OF STOCHASTIC SIGNALS}

It is known that the harmonic analysis of deterministic processes can be used to express them by means of Fourier series or Fourier integral depending on whether a periodic or aperiodic processes. In a similar way we can characterize the distribution of energy in stationary random processes using correlation analysis, respectively their internal structure and apply this procedure for needs of spectral analysis and optimization of combined dynamical systems dynamical properties.

We can express the relationship between the autocorrelation function $K x(\tau)$ of stationary stochastic process and its power spectral density $S x(\omega)$ (Levin, 1960) based on the WienerKhinchin relations. If the following conditions are met:

$$
\int_{-\infty}^{\infty} K_{x}(\tau) d \tau \leq M \quad \int_{-\infty}^{\infty} S_{x}(\omega) d \omega \leq N,
$$

where $\mathrm{M}$ and $\mathrm{N}$ are optional final.

Because the autocorrelation function is real and odd, if $\tau=0$, we can write:

$$
K_{x}(0)=D_{x}=\frac{1}{2 \pi} \int_{0}^{\infty} S_{x}(\omega) d \omega
$$

which is actually an expression of the overall standardized mean performance of the stationary stochastic signal. Similarly, we can express the spectral power density for $\omega=0$ :

$$
S_{x}(0)=2 \int_{0}^{\infty} K_{x}(\tau) d \tau
$$

We can see that the area under the autocorrelation function is proportional to the value of power spectral density for $\omega=0$.

Theory of white noise is applied to the combined dynamical systems, while the stochastic part of these systems approaching the normal probability distribution is represented as a process of constant and frequency unlimited intensity of power spectral density. Discoloration of white noise is expected to within the system. Using the normal probability distribution assumptions we can derive the Gaussian white noise intensity value, respectively value of combined dynamical systems stochastic part.

White noise is a stationary random process, in which the power spectral density is constant throughout the frequency range (Ondracek, 2003):

$$
S_{x}(\omega)=N=\text { const. }
$$

Autocorrelation function of white noise, whose mean power is infinite, by the Wiener-Khinchin relations is:

$$
K_{x}(\tau)=\frac{N_{0}}{2 \pi} \int_{-\infty}^{\infty} e^{j \omega \tau} d \omega=N \delta(\tau)
$$

Dispersion of frequency-limited white noise can be described by the intensity in the form (Besekerskij, 1969):

$$
D=\sigma^{2}=\frac{1}{2 \pi} \int_{-\omega_{m}}^{\infty_{m}} N d \omega=\frac{N \Delta \omega}{2 \pi}=N \Delta f
$$

Mean square error $\sigma$ is then equal to

$$
\sigma=\sqrt{N} \sqrt{\Delta f}
$$

the autocorrelation function for frequency limited white noise has form:

$$
K_{x}(\tau)=\frac{1}{\pi} \int_{0}^{\omega_{m}} N \cos \omega \tau d \omega=\frac{N}{\pi \tau} \sin \omega_{m} \tau
$$

For the normal probability distribution the dispersion of the stochastic process will be equal to $D=6 \sigma$. After substituting into equation (12) we obtain expression of random process intensity:

$$
N=\frac{D}{\Delta f}=\frac{6 \sigma}{\Delta f}
$$

\section{CONCLUSION}

To determine the $T_{P}$, time necessary for the execution of random events service routines in a given cycle, we are using the statistical evaluation of emerging events and their probability estimation. The white noise is a theoretical assumption - the colored noise is the input in practical terms. In our case, the system input in the form of stochastic events will be decolorized. It will assume generating of events underlying to the requirements of white noise (or frequency-limited white noise), while the color is transferred to the system itself.

\section{REFERENCES}

Banks, J. et al. (2001). Discrete-event system simulation, Prentice Hall, ISBN 0-13-088702-1, New Jersey

Besekerskij, V. A. (1969). Sbornik zadac po teorii automaticheskovo regulirovanja iI upravlenja, Nauka, pp. 588, Moscow

Levin, B. R. (1960). Teoria sluchajnych procesov i jijo primenenie z padiotechniky, Izdatelstvo Sovetskoe Radio, pp. 662, Moscow

Ondracek, O. (2003). Signaly a sustavy, Slovak University of Technology, ISBN 80-227-1875-0, Bratislava

Schreiber, P., Tanuska, P. (2007). Detection of the shortest robot trajectory by genetic algorithms, IN: DAAAM International Scientific Book, Vienna, ISSN 1726-9687, ISBN 3-901509-60-7, Katalinic, B. (Ed.), pp. 191-198, DAAAM International, Vienna

Stremy, M. (2010). Combined discrete dynamic systems, AlumniPress, ISBN 978-80-8096-113-8, Trnava

Zdansky, J.; Hrbcek, J. \& Simak, V. (2009). Using hybrid systems modelling to design a ventilation system in Road Tunnel, Proceedings of Technical computing Prague: 17th annual conference, Prague, ISBN 978-80-7080-733-0, Ausiello, G., Sannella, D. (Ed.), pp. 37 and CD, Humusoft, Prague 